\title{
Ultrasound features of deep-seated lipomas
}

\author{
Bhawan K. Paunipagar • James F. Griffith • \\ Darshana D. Rasalkar • L. T. C. Chow • S. M. Kumta • \\ Anil Ahuja
}

Received: 22 January 2010 / Accepted: 12 March 2010/Published online: 23 June 2010

(C) European Society of Radiology 2010

\begin{abstract}
Purpose The objective of this study was to describe the sonographic features of deep-seated lipomas.

Methods A retrospective review of the sonographic features of 64 deep seated lipomas in 64 patients (43 females, 21 males, mean age 46.5 , range $16-77$ years) seen over an 8-year period (1998-2006) was undertaken.

Results Features evaluated were location, size, shape, marginal definition, internal echogenicity, including the presence of intermingled muscle fibres and linear internal echoes, acoustic transmission and vascularity. Confirmation was histological in $37(58 \%)$ cases and by typical magnetic resonance imaging (MRI) appearance in 27 (42\%) cases. Conclusion The results show that although the features of deep-seated lipoma are more variable than those reported for subcutaneous lipomas, the presence of thin internal echoes in conjunction with other less specific features should enable a correct diagnosis.
\end{abstract}

Keywords Musculoskeletal $\cdot$ Soft tissue $\cdot$ Lipoma .

Deep seated · Ultrasound

B. K. Paunipagar $(\bowtie) \cdot$ J. F. Griffith · D. D. Rasalkar · A. Ahuja Department of Diagnostic Radiology and Organ Imaging,

The Chinese University of Hong Kong, Prince of Wales Hospital, Shatin, Hong Kong, SAR, China

e-mail: drbhawan@cuhk.edu.hk

\section{T. C. Chow}

Department of Anatomical and Cellular Pathology,

The Chinese University of Hong Kong, Prince of Wales Hospital,

Shatin, Hong Kong, SAR, China

\section{S. M. Kumta}

Department of Orthopedics and Traumatology, The Chinese

University of Hong Kong, Prince of Wales Hospital,

Shatin, Hong Kong, SAR, China

\section{Introduction}

Soft-tissue lipomas are the most common type of benign mesenchymal tumours made up of mature adipose tissue [1, 2]. These tumours can occur at any age, but are most common in the fifth or sixth decade of life, and are multiple in $5 \%$ of patients $[3,4]$, often associated with a period of weight gain. They tend to stabilize in size after an initial period of steady growth. Lipomas are usually relatively small with diameters of about $1-3 \mathrm{~cm}$, but in rare cases they can grow over several years into "giant lipomas" that are $10-20 \mathrm{~cm}$ across and weigh up to $4-5 \mathrm{~kg}[5,6]$.

Soft tissue lipomas are classified by location into superficial and deep lesions. Superficial lipomas occur in the subcutaneous tissues, while deep lipomas occur deep to the investing fascia (i.e. subfascial). Superficial lipomas are commonly found in the posterior trunk, neck and proximal extremities [6]. Clinical as well as ultrasound appearances of superficial lipomas are characteristic, and they usually can be diagnosed clinically. Ultrasound is performed if there is doubt regarding the clinical diagnosis or to confirm the extent of tumour.

Deep-seated soft-tissue lipomas are less common than superficial lipomas [6] and may be located above muscle (supramuscular), between muscle (intermuscular), within muscle (intramuscular) or below muscle (submuscular). Lipomas occurring within nerves or tendons sheaths or bone are generally considered as distinct, separate entities. Deep-seated lipomas usually occur in the extremities and do not have as characteristic a clinical presentation as subcutaneous lipomas. They tend to be firmer in consistency, less well-defined and often mimic a sarcoma [7, 8]. The ultrasound features of deep-seated lipomas have been noted in case reports and or grouped together with superficial lipomas [9]. A specific comprehensive description of the 
ultrasound features of deep-seated lipomas has not been reported. We believe that certain ultrasound features of subfascial lipomas are constant and quite characteristic. Knowledge of these characteristic ultrasound features would facilitate early recognition of deep-seated lipomas and expedite investigation.

\section{Materials and methods}

A retrospective review of the ultrasound features of 64 deep-seated lipomas in 64 patients examined in a single institution over an 8-year period between March 1998 and April 2006 was performed. After institutional ethic approval, all ultrasound examinations were performed using either 10-7 or 13-5 MHz linear array transducers (Sonoline Elegra Advanced, Siemens, Erlangen, Germany) by one of three radiologists experienced in musculoskeletal ultrasound. Colour Doppler analysis was routinely performed. Specific features specifically evaluated were the site of the tumour, location within the muscle compartment, size, shape, and marginal definition, internal echogenicity, including the presence of linear internal echoes, acoustic attenuation and vascularity. Location was categorised as supramuscular, intermuscular, intramuscular or submuscular. Shape was categorised as roundish, oblong, fusiform or geographical. Margin definition was either well-defined or ill-defined. Acoustic transmission was categorised as being greater than, equal to or less than adjacent musculature. Internal echogenicity was either hypo-, iso- or hyperechoic to adjacent muscle. Linear internal echoes were noted defined as being either fine or coarse. Orientation of internal echoes relative to the long axis of the tumour was also noted. Tumour vessel density or vascularity was categorised as high, moderate or low vascularity.

Following ultrasound examination, magnetic resonance imaging (MRI) examination was performed in 54 (84.3\%) cases. The diagnosis of subfascial lipoma was established by excision biopsy in $37(58 \%)$ of 64 cases. In the remaining $27(42 \%)$ cases, the final diagnosis was established on the basis of typical MR appearances and clinical follow-up (for a mean period of 4.5 months, range 3-7 months).

\section{Results}

Sixty-four deep-seated lipomas were seen in 64 patients, comprising 43 females and 21 males, with a mean age of 46.5 years (range 16-77 years). The location and distribution of the 64 deep-seated lipomas is summarised in Table 1 and the pie chart (Fig. 1). Of the 64 deep-seated lipomas, three $(5 \%)$ were supramuscular, $17(27 \%)$ were intermus-
Table 1 Summary of the location and distribution of the deep-seated lipomas.

\begin{tabular}{lll}
\hline $\begin{array}{l}\text { General site of } \\
\text { involvement }\end{array}$ & $\begin{array}{l}\text { More specific site } \\
\text { of involvement }\end{array}$ & Frequency \\
\hline Thorax $(n=14)$ & Anterior chest wall & 1 \\
& Dorsal and scapular regions & 13 \\
Abdomen $(n=2)$ & Anterior abdominal wall & 0 \\
& Lumbar and loin region & 2 \\
Upper limb $(n=25)$ & Shoulder girdle & 8 \\
& Flexor compartment arm & 5 \\
& Extensor compartment arm & 2 \\
& Flexor compartment forearm & 9 \\
& Extensor compartment forearm & 1 \\
& Pelvic girdle & 4 \\
Lower limb $(n=23)$ & Extensor compartment thigh & 7 \\
& Flexor compartment thigh & 8 \\
& Adductor compartment thigh & 2 \\
& Extensor compartment thigh & 1 \\
& Flexor compartment thigh & 0 \\
& Peroneal compartment thigh & 1 \\
& & 64 \\
\hline
\end{tabular}

cular, $36(56 \%)$ were intramuscular and five (7\%) submuscular. Maximum diameter ranged from $1 \mathrm{~cm}$ to $15 \mathrm{~cm}$. Shape was roundish in $29(45 \%)$, ovoid in seven (11\%), oblong in $20(31 \%)$, and geographical in eight $(12 \%)$. Margin was well-defined in $50(78 \%)$ and ill-defined $14(22 \%)$. Overall lesion echogenicity was hypoechoic to muscle in 14 (22\%), isoechoic to muscle in $13(21 \%)$ and hyperechoic to muscle in 37 (57\%) (Fig. 2a-c). Acoustic transmission compared with adjacent muscle was increased in 24 (37.5\%) (Fig. 3), equal in 18 (22.1\%), and decreased in 22 (33.4\%). Compared with smaller size lipomas, the larger

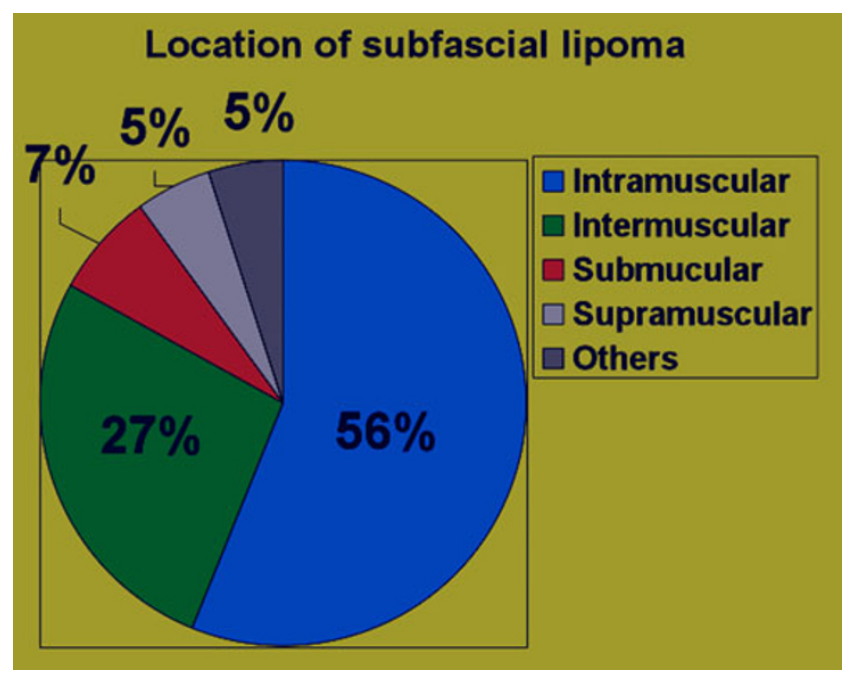

Fig. 1 Pie chart showing the distribution of the subfascial lipomas. 


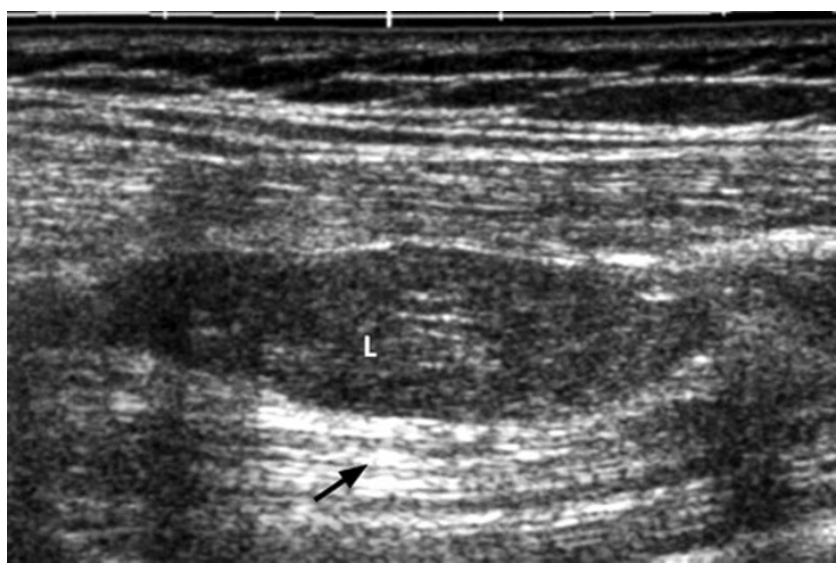

Fig. 2a-c Grey-scale, panoramic ultrasound view demonstrating different echogenicities of the subfascial lipomas $(L)$ relative to the echogenicities of the adjacent muscles. a Hyperechoic; b hypoechoic; c mixed echogenicity relative to adjacent muscles.

ones showed a greater degree of acoustic transmission relative to muscle. The linear internal echoes were fine in appearance, were of three types (long continuous, medium sized discontinuous or whorled/onion peel) and were orientated parallel to the long axis of the tumour in 63 (98\%) of 64 deep-seated lipomas (Fig. 4a-c). Twenty (31\%) lipomas revealed mild internal vascularity, eight (12\%) minimal internal vascularity and $36(57 \%)$ no internal vascularity. In all cases, deep-seated lipoma was reported as the most likely diagnosis at ultrasound examination.

\section{Discussion}

As opposed to subcutaneous lipomas, deep-seated lipomas cannot usually be diagnosed clinically. Not infrequently, the clinical presentation is similar to that of a sarcoma [7,8]. Ultrasound is usually the first line of investigation.

Epidemiologically and histologically, deep-seated lipomas are very similar to subcutaneous lipomas. They both tend to occur in middle-aged patients, tend to be more common in females and tend to present following a period of weight gain. They stabilise in size after a period of growth, usually varying from months to a couple of years. Both subcutaneous and deep-seated lipomas are comprised of mature fat cells. Cartilage and bone may occasionally be seen within lipomas, particularly in longstanding disease.

Most deep-seated lipomas tend to be roundish or oblong in shape, as opposed to subcutaneous lipomas which tend to be ovoid [2]. Similar to subcutaneous lipomas, many of the ultrasound features of deep-seated lipomas tend to be variable. In $60 \%$ [4] to $88 \%$ [2] of cases, subcutaneous lipomas tend to be well-defined, while $29 \%$ [4] to $76 \%$ [2] are hyperechoic to muscle. Classically, lipomas have been described as being homogeneous and hyperechoic, but there are frequent exceptions [10]. An acoustic impedance mismatch exists at fat-water and water-fat interfaces [11]. Since pure fatty tumours have few interfaces and less of an acoustic impedance mismatch, they appear echo-free, whereas lipomas with a mixed cellularity have an increased number of interfaces and appear echogenic. Altered acoustic transmission resulting in either posterior enhancement or attenuation is not a feature of subcutaneous lipomas. This is to be expected, since the tissue is comparable with surrounding fat. Conversely, acoustic transmission in deep-seated lipomas tends to be increased, particularly in the larger lesions since the acoustic attenuation of fat is less than that of skeletal muscle. Of deepseated lipomas, 98\% contained fine internal echoes parallel to the long axis of the tumour. Deep-seated lipomas are recognised to be more vascular than subcutaneous lipomas,
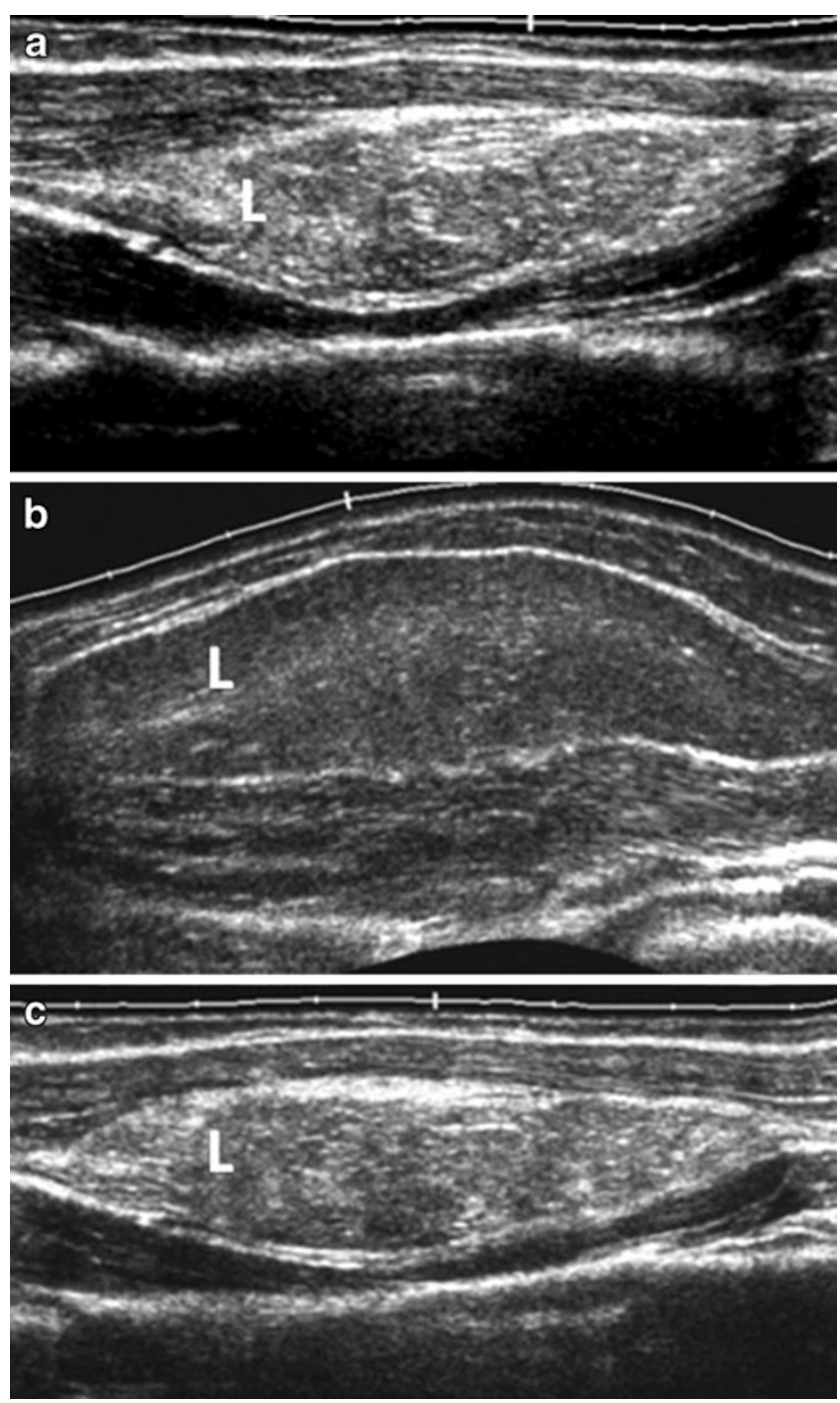

Fig. 3 Grey scale ultrasound of subfascial lipoma. Note the increased acoustic transmission (black arrow). 
Fig. 4a-c Grey-scale ultrasound images showing three different types of the fine, internal, echo pattern parallel to the long axis of lesion, as shown in the companion line diagrams. a Long continuous internal echos; traversing the entire length of the lipoma without disruption. b Medium-sized, discontinuous. Note the broken appearance of the internal echoes. c Whorled or onion peel. These are continuous but are circular and arranged in layers.

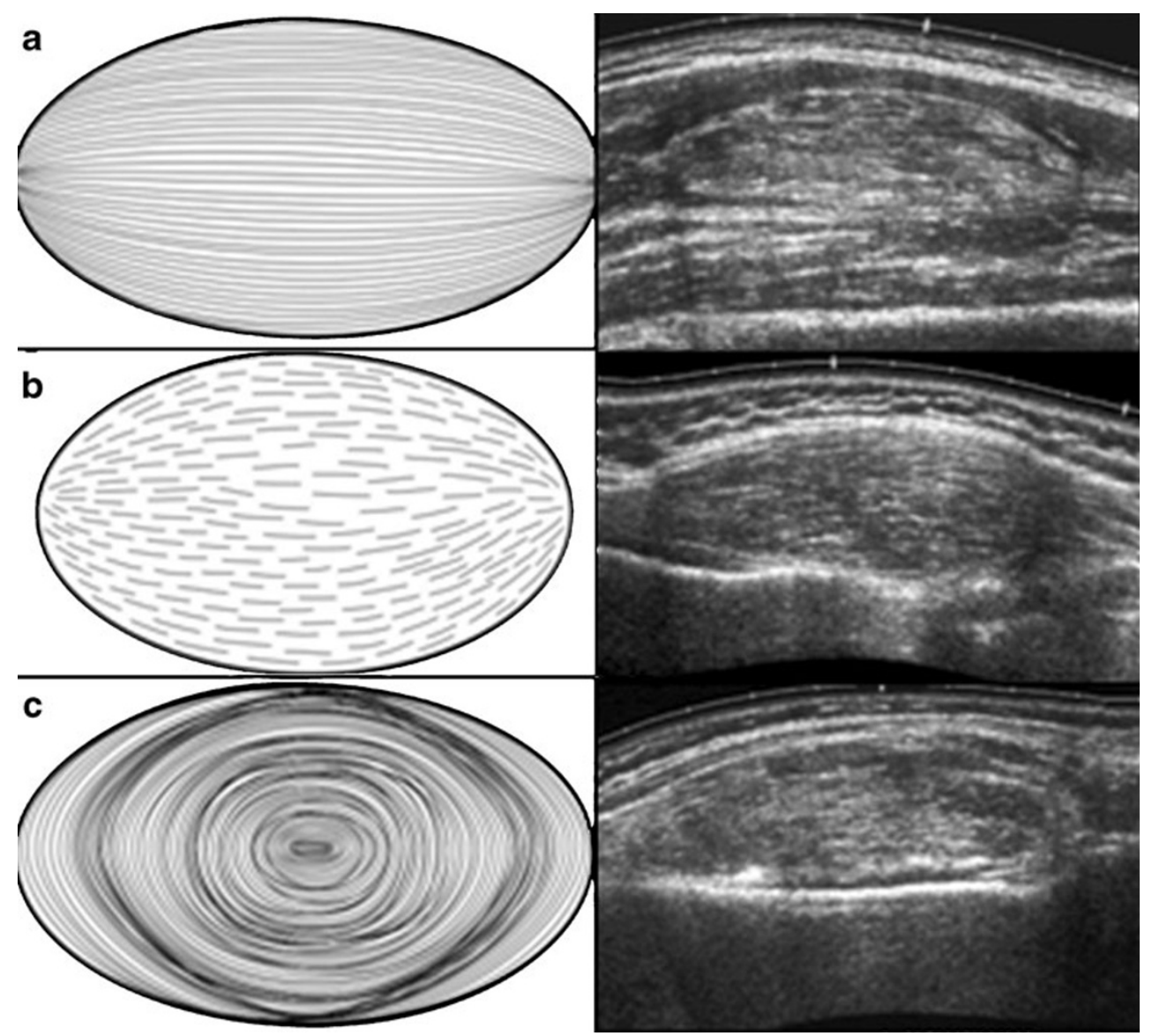

a feature reflected in the minimal to mild internal vascularity evident in $43 \%$ of tumours.

Differentiation of lipomas containing non-adipose areas from that of well-differentiated liposarcoma may at times be difficult. Features that suggest malignancy include increased patient age (greater than 60 years), large lesion size $(>10 \mathrm{~cm})$, gender (male); presence of thick septa $(>2 \mathrm{~mm})$, presence of nodular and/or globular or nonadipose mass-like areas, and decreased percentage of fat composition (less than 75\% fat) [7].

These differentiating features are mainly based on crosssectional studies such as computed tomography (CT) and MRI, and the demonstrable intrinsic thin septa were $<2 \mathrm{~mm}$ in about 37-49\% of lipomas [7, 13-16]. Well-differentiated liposarcomas have this feature only rarely (4-9\% of cases) $[12,13]$. In addition, Ohguri et al. [13] showed that the septal enhancement pattern on contrast material-enhanced MR images may be helpful in distinguishing lipoma from welldifferentiated liposarcoma. In their study, 58\% of lipomas with septa showed no enhancement and 37\% revealed moderate enhancement [13]. Well-differentiated liposarcomas showed moderate $(25 \%)$ to marked $(75 \%)$ enhancement of the septa in all cases [13]. No differentiation was made in enhancement between lesions with thin $(<2 \mathrm{~mm})$ versus thick
( $>2 \mathrm{~mm}$ ) septa [13]. Therefore, soft-tissue lipomatous lesion with only thin septa that do not enhance at MRI can be confidently diagnosed as a lipoma [11].

Thick septa $(>2 \mathrm{~mm})$ as well as nodular or globular regions of non-adipose tissue are reported in $28-31 \%$ of cases of soft-tissue lipomas [7, 12-15]. Such lipomas cannot be distinguished from well-differentiated liposarcoma with imaging alone, and biopsy is mandatory to exclude well-differentiated liposarcoma and the possibility of dedifferentiation [17]. These non-adipose regions likely correspond to previously described mesenchymal elements or areas of fat necrosis.

Subcutaneous and intermuscular lipomas frequently demonstrate a fibrous capsule with attenuation similar to that of muscle at CT and low signal intensity on all MR images regardless of pulse sequence. This capsule is not seen with intramuscular lesions, as it cannot be distinguished from the surrounding muscle. Intramuscular lipomas may also demonstrate irregular margins and interdigitations with skeletal muscle that create a striated appearance [18]. This feature has not been described with liposarcoma and allows confident diagnosis of intramuscular lipoma. Intermuscular lipomas, particularly those centred in the popliteal or inguinal region may encase the 
neurovascular bundle. This feature limits the ability to perform complete resection and increases the likelihood of local recurrence.

The ultrasound features of deep-seated lipomas have been noted in case reports and/or grouped together with superficial lipomas [9]. A specific comprehensive description of the ultrasound features of deep-seated lipomas has not been reported.

The other differential diagnosis of deep-seated lipomas includes nerve sheath tumour, giant cell tumour of tendon sheath, haemangioma and haematoma. These are relatively easier in correlation with the clinical history (age, onset, etc.), based on imaging features, and associated findings like anatomical site and vascularity; phleboliths and serial change on imaging follow-up often give additional clues.

Complete resection of deep-seated lipomas is often difficult, and the postoperative recurrence rate is $3-62 \%$. In fact, incomplete resection is favoured because development of muscle dysfunction due to fatty infiltration is gradual, and the patient tends to compensate well through recruitment of other muscles. By comparison, the rapid loss of function secondary to the surgical removal of a muscle is, in general, poorly tolerated.

There are some limitations of this study. One of the major limitations is that this retrospective study includes benign lipomas and there is no comparison with other solid deep-seated masses. Histological confirmation is available for $37(58 \%)$ cases, whereas in other $27(42 \%)$ cases confirmation is mainly by typical MRI appearance, with overall mean clinical follow-up for most lesions 3-7 months, so that potential slow-growing, well-differentiated liposarcomas could be ignored. These are probably intrinsic problems, as often many asymptomatic patients are usually unwilling to undergo invasive procedures, and also these patients often have low incidence of longer mean follow-up in the absence of significant complaints or complications. Nevertheless, this study demonstrates specific imaging features (fine internal echopattern parallel to the long axis of lesion and posterior acoustic enhancement) and has a diagnostic value for deepseated lipomas. The remainder of the lesions which lack these typical features, on the contrary, should be complemented by additional imaging (MRI) or biopsy.

In conclusion, this study is of potential interest for future ultrasound-related research on deep-seated lipomas and provides characteristic ultrasound features to facilitate early recognition and expedite investigation of deep-seated lipomas.

\section{References}

1. Bancroft LW, Kransdorf MJ, Peterson JJ, O'Connor MI (2006) Benign fatty tumors: classification, clinical course, imaging appearance, and treatment. Skeletal Radiol 35(10):719-733

2. Ahuja AT, King AD, Kew J, King W, Metreweli C (1998) Head and neck lipomas: sonographic appearance. AJNR Am J Neuroradiol 19(3):505-508

3. Salam GA (2002) Lipoma excision. Am Fam Physician 65 (5):901-904

4. Fornage BD, Tassin GB (1991) Sonographic appearances of superficial soft tissue lipomas. J Clin Ultrasound 19(4):215-220

5. Rydholm A, Berg NO (1983) Size, site and clinical incidence of lipoma. Factors in the differential diagnosis of lipoma and sarcoma. Acta Orthop Scand 54(6):929-934

6. Terzioglu A, Tuncali D, Yuksel A, Bingul F, Aslan G (2004) Giant lipomas: a series of 12 consecutive cases and a giant liposarcoma of the thigh. Dermatol Surg 30(3):463-467

7. Kransdorf MJ, Bancroft LW, Peterson JJ, Murphey MD, Foster WC, Temple HT (2002) Imaging of fatty tumors: distinction of lipoma and well-differentiated liposarcoma. Radiology 224(1):99-104

8. Al-Qattan MM, Weinberg M, Clarke HM (1995) Two rapidly growing fatty tumors of the upper limb in children: lipoblastoma and infiltrating lipoma. J Hand Surg Am 20(1):20-23

9. Inampudi P, Jacobson JA, Fessell DP, Carlos RC, Patel SV, Delaney-Sathy LO et al (2004) Soft-tissue lipomas: accuracy of sonography in diagnosis with pathologic correlation. Radiology 233(3):763-767

10. Lin J, Fessell DP, Jacobson JA, Weadock WJ, Hayes CW (2000) An illustrated tutorial of musculoskeletal sonography: part I, introduction and general principles. AJR Am J Roentgenol 175 (3):637-645

11. Murphey MD, Carroll JF, Flemming DJ, Pope TL, Gannon FH, Kransdorf MJ (2004) From the archives of the AFIP: benign musculoskeletal lipomatous lesions. Radiographics 24(5):14331466

12. Kransdorf MJ, Moser RP Jr, Meis JM, Meyer CA (1991) Fatcontaining soft-tissue masses of the extremities. Radiographics 11 (1):81-106

13. Ohguri T, Aoki T, Hisaoka M et al (2003) Differential diagnosis of benign peripheral lipoma from well-differentiated liposarcoma on MR imaging: is comparison of margins and internal characteristics useful? AJR Am J Roentgenol 180:1689-1694

14. Ha TV, Kleinman PK, Fraire A et al (1994) MR imaging of benign fatty tumors in children: report of four cases and review of the literature. Skeletal Radiol 23:361-367

15. Hosono M, Kobayashi H, Fujimoto R et al (1997) Septum- like structures in lipoma and liposarcoma: MR imaging and pathologic correlation. Skeletal Radiol 26:150-154

16. Gaskin CM, Helms CA (2004) Lipomas, lipoma variants, and well-differentiated liposarcomas (atypical lipomas): results of MRI evaluations of 126 consecutive fatty masses. AJR Am J Roentgenol 182:733-739

17. Kransdorf MJ, Meis JM, Jelinek JS (1993) Dedifferentiated liposarcoma of the extremities: imaging findings in four patients. AJR Am J Roentgenol 161:127-130

18. Kransdorf MJ, Murphey MD (1997) Imaging of soft tissue tumors. Saunders, Philadelphia, pp 57-101 